\title{
New high-speed system for controlling the parameters of a nuclear reactor in a nuclear power plant
}

\author{
Roman Davydov ${ }^{1, *}$, Valery Antonov ${ }^{1}$, Sergey Makeev ${ }^{1}$, Yury Batov ${ }^{1}$, Valentin Dudkin $^{2}$, and Nikita Myazin ${ }^{1}$ \\ ${ }^{1}$ Peter the Great St. Petersburg Polytechnic University, St. Petersburg, Russia \\ ${ }^{2}$ The Bonch-Bruevich Saint Petersburg State University of Telecommunications, St. Petersburg, Russia
}

\begin{abstract}
The necessity of modernizing current control systems for functional units of a nuclear power plant, as well as the development of new control systems with a high degree of reliability and speed, is substantiated. The advantages of using optical sensors and fiber-optic communication lines to solve these problems are noted. Cases for which it is necessary to develop new fiber-optic sensors for monitoring parameters, for example, the flow of coolant or feed water, are considered. In some of them, it is more expedient to use standard designs of fiber-optic sensors to control the operating parameters of various blocks, for example, to control the electric field strength. A device and a control scheme for the parameters of the units and systems of a nuclear power plant using fiber-optic communication lines have been developed. The results of measuring various parameters of a nuclear reactor are presented. They showed that our proposed fiber-optic control and monitoring system for nuclear power plants operates more reliably and efficiently than systems with analogue control and measurement channels. The use of fiberoptic systems allows real-time remote control and high-speed control in terms of issuing commands to devices. This is very important when servicing a nuclear power plant while it is operating in extreme conditions.
\end{abstract}

\section{Introduction}

In the modern world, every year for the sustainable development of society requires more and more electric energy [1-14]. One of the most promising ones, both with an environmental component (insignificant increase in the environmental load) and for large capacities for large enterprises and cities, is nuclear energy [6-9, 1524]. When operating nuclear power plants (NPPs) of various capacities, several tasks arise. One of them is the creation of control and monitoring systems that ensure the stable operation of equipment operating in continuous mode at nuclear power plants [25-32]. And to provide reliable remote control of various systems and units of the station, for example, when the station is decommissioned, or in the "conservation" mode of a nuclear reactor [6, 9, 23-26]. The greatest difficulties arise in the development of these systems for nuclear power plants of medium and low power. When developing systems for nuclear power plants of medium and low power, difficulties arise due to the peculiarities of their operation - such stations can be located on mobile facilities, where both the space for them and the number of staff are limited [6, 9, 19-22].

It should also be noted that the control and monitoring systems must work reliably for a certain time in a complex emergency at a nuclear power plant (one or more parameters, for example, radiation level or temperature, reach maximum permissible values or exceed them) [6, 9, 23-28, 33]. The operational experience of various nuclear power plants has shown that the monitoring and diagnostics systems of nuclear installations, based on electronic devices, often fail for various reasons. Particularly, due to the compact placement of all systems (a large number of interference and interference of various types, sudden changes in temperature, etc.). Especially negatively affect the electronic devices and their connecting elements (connectors, cables, etc.) powerful surges of voltage and current when starting various mechanisms [9, 23-28, 34]. As well as sudden triggering of key elements and protection relays in transformer substations of a moving object. This creates big problems when using mobile devices, cable lines with analog signals and digital systems [23-28, 32-38]. Modern shielding systems cannot remove the noise that is generated in the control signals. This leads to equipment malfunctions and unauthorized shutdowns of reactor support systems. In this case, control over the operation of the reactor installation may be lost, which means loss of control of the nuclear power plant.

Various designs of optical sensors with laser radiation, as well as fiber-optic communication lines (FOCL) for transmitting information are immune to

\footnotetext{
* Corresponding author: davydovroman@outlook.com
} 
electromagnetic interference [38-45]. Especially to those that occur during sudden power surges. Besides, the use of optical fiber allows galvanic isolation between different systems in many devices (for example, at NPPs these are monitoring systems. These devices have increased flexibility and can easily be placed in hard-toreach places. Examples of applications of these devices in the construction of complex facilities [46-49], proved the viability of their use. So, the development of parameter control systems based on fiber-optic communication lines for nuclear power plants is an urgent task. We present one of its possible solutions for nuclear power plants with a VVER-1000 reactor (for other types of reactors, it will be necessary to make some improvements and changes to the monitoring and control system we developed).

\section{Materials and methods}

We have established key features in the development of fiber-optic systems for monitoring and control of the NPPs, considering the specifics of their operation. The most important of them is ensuring the speed and reliability of information transfer to large volumes for making correct and informed decisions, especially at the final stage of the life cycle of reactor plants. This is a rather difficult period in the operation of the plant and in the operation of the NPP there are already a number of negative factors: a high degree of wear of the main equipment, a change in the generation of maintenance personnel (they did not participate in the commissioning of the reactor), etc. Without operational data on the life cycle of a nuclear power plant, it is quite difficult to decommission it [9, 19-26].

In Fig. 1 shows a sectional view of a design of a VVER-1000 reactor. Its main blocks and units are designated. Their operation parameters must be monitored continuously or controlled with the receipt of confirmation signals about the execution of specified procedures. From these units and other reactor nodes, the central computer of the NPP must receive reliable information about the parameters of the reactor and the execution of the given commands. The complex placement of blocks and other nodes in the design of the reactor, temperature differences and the presence of a large number of interferences require the development of a certain configuration of optical control and monitoring systems. Studies have shown that it is most advisable to use separate optical channels: for measurements, for monitoring parameters, for transmitting control commands and confirming their execution.

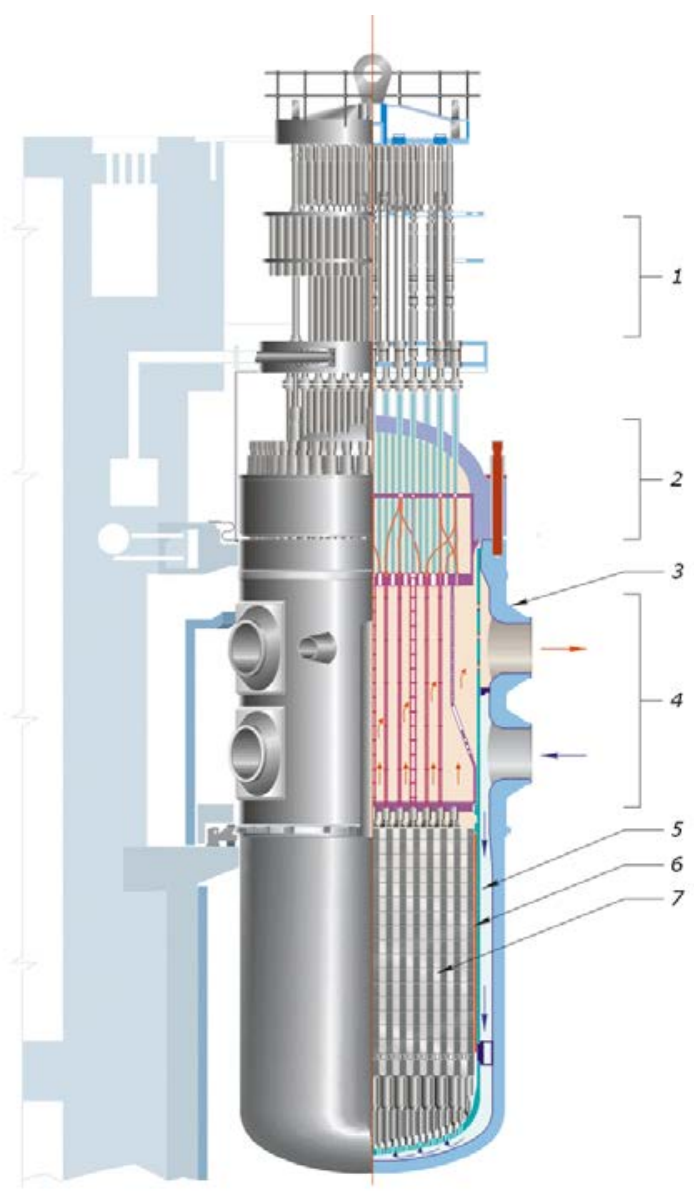

Fig. 1. The design of the reactor VVER-1000: 1 - control and protection system drives; 2 - reactor cover; 3 - reactor vessel; 4 - a block of protective pipes; 5 - mine; 6 - partition of the core; 7 - fuel assemblies, control rods.

In Fig. 2 presents a block diagram of a fiber-optic system that we developed. The use of optical sensors and fibers has led to a decrease in the area occupied by information transmission lines and measuring elements. The number of channels for transmitting information, control commands, and measurements was increased. Each channel uses a single-mode fiber, which is more resistant to various influences than the multimode used earlier in nuclear power plants. The presence of extra free space in the communication line placement system allows creating two more backup channels for each active channel. Besides, various converters and amplifiers were excluded from the design of measuring devices, which, being located in the radial zone of the reactor, were subject to numerous interferences and required additional protection [19-22, 35-38].

During the measurements, all electronic circuits and optical devices (lasers, photodetectors, modulators) are placed outside the radial zone of the reactor. They can be located at a distance of more than $200 \mathrm{~m}$ from the reactor. Only fiber-optic sensors and optical fiber are located in the reactor zone. This fundamentally distinguishes the developed design from previously used systems. Information on the channel is transmitted only in one direction. This eliminates additional interference when switching optical elements. 


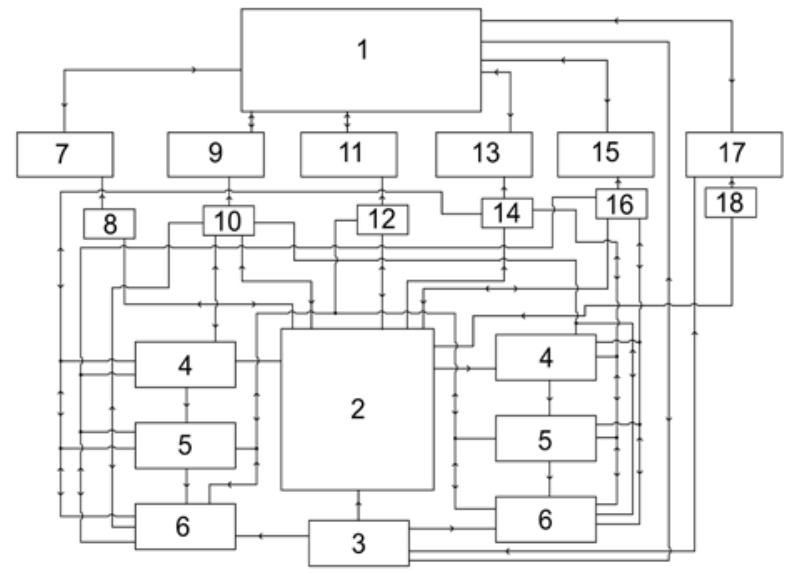

Fig. 2. A block diagram of control and monitoring of NPP operating parameters based on FOTS and optical sensors: 1 NPP central computer, 2 - reactor, 3 - reactor emergency shutdown system, 4 - turbine, 5 - electrical energy converter, 6 - transformer substation with key elements, 7 - flow rate and feedwater flow control unit, 8 - fiber optic flow control sensors, 9 - pressure control unit in various reactor systems, 10 - fiber optic pressure sensors, 11 - magnet control unit, 12 - fiber-optic induction sensors of the magnetic field, 13 - control unit of the reactor and substation systems to compensate for the voltage of the electric field, 14 - fiber optic sensor of the electric field strength, 15 - temperature control unit, 16 - fiber-optic temperature sensors , 17 - ionizing radiation control unit, 18 fiber-optic sensors for changing the level of ionizing radiation.

The new principle of placement of control units and measuring systems, as well as the use of fiber optic links significantly increases the reliability of the entire system, unlike previously developed ones. Besides, the use of fiber-optic communication lines made it possible to use rectangular pulses of various durations for transmitting commands to various control systems. In previously used analog systems, the shape of the pulses was distorted. This especially negatively affects the reliability of the execution of control commands.

\section{Results and discussion}

The central measuring elements in the system are fiberoptic sensors. Today, the conceptual foundations of many fiber-optic sensors are well developed and various industrial designs of them are used (measuring humidity, pressure, temperature, magnetic field induction and electric field strength) are produced. The accuracy of measurements of physical parameters and the reliability of these sensors corresponds to the concept of a fiberoptic system that we developed.

As an example, the work presents the optical sensors developed by us (correlation flowmeter) for measuring the flow rate $\mathrm{q}$ of coolant and feed water. A sensor for determining the radiation dose will be considered in the following works of the authors. The remaining types of optical sensors that are proposed to be used in the developed fiber optic system are standard. In Fig. 3 shows its structural diagram of the design of the correlation flowmeter we developed using an annular fiber-optic detector for recording $\gamma$ - quanta.

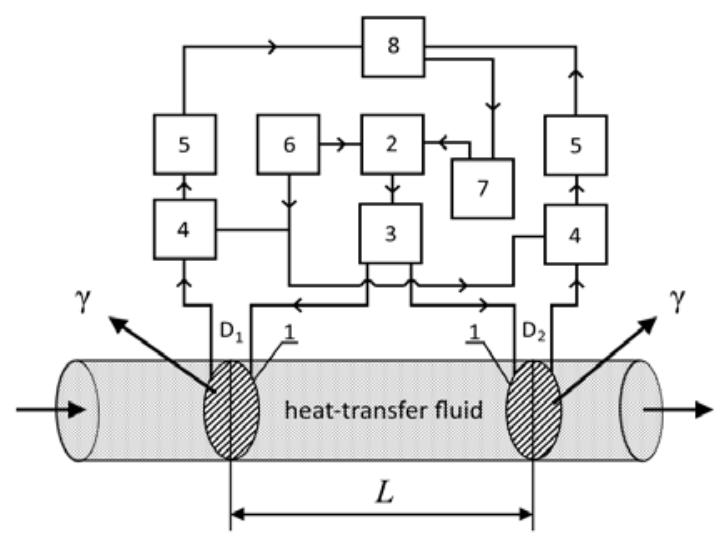

Fig. 3. Block diagram of an optical device for measuring the flow of a liquid medium in a pipeline. 1 - ring fiber-optical system, 2 - laser transmitting module, 3 - optical divider, 4 photoreceiver module, 5 - analog-digital converter, 6 - power supply, 7 - pulse generator, 8 - processing device and control.

The coolant in the reactor core is irradiated with $\gamma$ radiation. After leaving the core, the coolant (for example, liquid sodium or lead melt with bismuth), flowing through the pipeline emits $\gamma$-quanta. The radiation intensity of $\gamma$-quanta as it flows through the pipeline decreases in proportion to a certain dependence (it is determined experimentally for each coolant).

Under the influence of $\gamma$-quanta with a single-mode optical fiber, the intensity of the laser signal propagating through it changes. From the two ring sensors located at a certain distance L (Fig. 3), changes in the intensity of laser radiation are recorded. The fiber-optic system that we developed allows us to arrange a large number of these optical pairs of sensors in various sections of the pipeline. The data obtained earlier determine the dependence of the attenuation of the intensity (number) of $\gamma$-quanta emitted by the medium on time. The experiments showed that for measurements of $q$ it is most advisable to use laser radiation in the form of short pulses with a duration of $0.1 \mathrm{~ms}$ to $10 \mathrm{~ms}$ (depending on the flow rate of the coolant and its composition).

Ten pairs of fiber-optic ring sensors with $\mathrm{L}=10 \mathrm{~m}$ are used. Sensors are located at $20 \mathrm{~m}$ from each other. In Fig. 4 shows the work of the ring fiber-optic sensor. In section $\mathrm{D}_{1}$ and $\mathrm{D}_{2}$, an optical fiber wound around a pipeline is affected by a different number of $\gamma$-quanta. The refractive index of the fiber in zones $D_{1}$ and $D_{2}$ changes. Laser radiation passing through these zones has different intensities. By changing the dependence of the amplitude of the recorded laser radiation on various pairs of sensors, the flow time of the coolant between the sensors $\Delta \mathrm{t}$ is determined. The error in determining the flow rate $\mathrm{q}$ in the primary reactor using the sensor developed by us is less than $2 \%$. The measurement result q coincided with the results of measuring the NMR flow by a flowmeter with a measurement error of less than $1 \%$ $[32,50]$. It should be noted that the NMR flowmeter, in contrast to the proposed optical sensor, is a very large device that requires special maintenance [32, 50]. Only one such device can be placed in the design of a low power nuclear power reactor. This is not enough for effective control. 


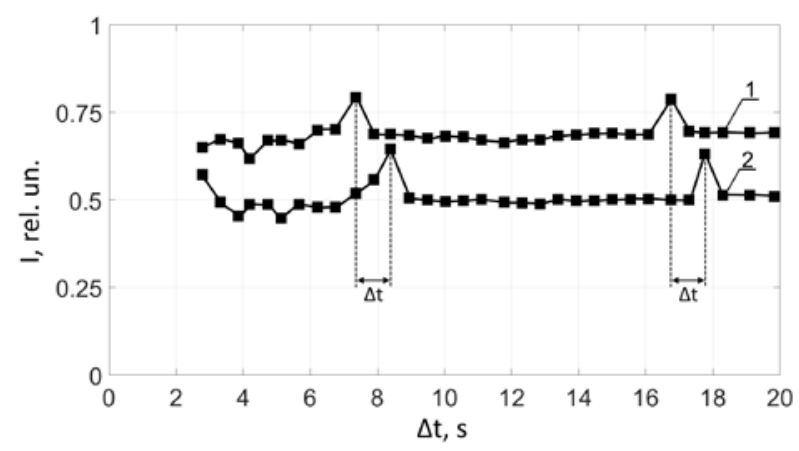

Fig. 4. The dependence of the intensity of the recorded laser radiation on the flow time of the coolant between the sensors. Graph 1 corresponds to measurements in section $\mathrm{D}_{1}$, graph 2 in section $\mathrm{D}_{2}$.

The principle of operation of ring optical sensors for measuring the flow rate of feed water is almost the same as for sensors measuring $\mathrm{q}$ coolant. Several pairs of sensors are used for measurements. The radiation emitted during the decay of $16 \mathrm{~N}$ nuclei contained in the feed water is detected, and changes in the intensity of $\gamma$ quanta are recorded at shorter distances.

An analysis of the results of measuring the flow of feed water showed that the measurement error q was within $2 \%$. For smaller values of the measured values, the error also turns out to be smaller, which is associated with the features of the principle of operation of the ring fiber-optic detector.

\section{Conclusion}

The results showed that our proposed fiber-optic control and monitoring system for nuclear power plants operates more reliably and efficiently than systems with analog control and measurement channels.

A large number of signal processing systems (including complex ones) can be used in the developed system. All devices for processing and generating optical signals are located at a considerable distance from the reactor behind the protective shields, since the signal attenuation in the fiber is $0.1 \mathrm{~dB} / \mathrm{km}$. When using analog signal data processing, this will lead either to an increase in the number of modules used or to a weakening of the useful signal with an increase in the transmission distance. The reliability of the control and management system will be significantly reduced.

The use of fiber-optic systems allows real-time remote control and high-speed control in terms of issuing commands to various mechanisms and devices. This is very important when servicing a nuclear power plant while it is operating in extreme conditions.

\section{References}

1. T. Fejling, E. Torosyan, O. Tsukanova, O. Kalinina, IOP Conference Series: Materials Science and Engineering 497 (1), 012027 (2019)
2. V. Vilken, O. Kalinina, S. Barykin, E. Zolotova, IOP Conference Series: Materials Science and Engineering 497(1), 012037 (2019)

3. O. Kalinina, E. Balchik and S. Barykin, MATEC Web of Conference 239, 04021 (2018)

4. V. Vilken, O. Kalinina and A. Dubgorn, E3S Web of Conference 33, 03012 (2018)

5. A. Bril, O. Kalinina and A. Levina, E3S Web of Conference,33, 03004 (2018)

6. N.D. Agafonova, M.Y. Egorov, V.V. Sergeev, M.A. Gotovskii, P.A. Kruglikov, M.E. Lebedev, A.V. Sudakov, E.D. Fedorovich and B.S. Fokin, Atomic Energy 123(3), 154-158 (2018)

7. J. Kaikko, A. Mankonen, E. Vakkilainen and V. Sergeev, Energy Procedia 120, 572-579 (2017)

8. M. Petrichenko, V. Sergeev, E. Kotov, D. Nemova and D. Tarasova, Advances in Intelligent Systems and Computing 983, 839-848

9. V.E. Fortov and A.A. Makarov, Physics-Uspekhi 52(12), 1249-1265 (2009)

10. V.V. Davydov, Russian Physics Journal 42(9), $822-$ 825 (1999)

11. V.I. Antonov, V.L. Badenko, R.V. Davydov, V.I. Maslikov and D.V. Molodtsov, Journal of Physics: Conference Series 1236(1), 012049 (2019)

12. R.V. Davydov, V.I. Antonov and D.V. Molodtsov, Journal of Physics: Conference Series 1135(1), 012088 (2018)

13. R. Davydov, V. Antonov, D. Molodtsov, A. Cheremisin and V. Korablev, MATEC Web of Conf. 245, 15002 (2018)

14. R. Davydov, V. Antonov, D. Molodtsov and A. Trebukhin, Advances in Intelligent Systems and Computing 692, 915-920 (2018)

15. A.L. Sirotkina, E.D. Fedorovich and V.V. Sergeev, High Temperature 56(5), $732-737$ (2018)

16. T.J.B. Taweel, E. Sokolova, V. Sergeev, D.B. Solovev, IOP Conference Series: Materials Science and Engineering 463(1), 032101 (2018)

17. O.V. Novikova, A.N. Grishki, I.S. Khrebetenko and N.A. Yudina, IOP Conference Series: Earth and Environmental Science 288(1), 012065 (2019)

18. T. Bugaeva, A. Khabarov, O. Novikova and U. Plotkina, IOP Conference Series: Materials Science and Engineering 497(1), 012056 (2019)

19. V.V. Davydov, V.I. Dudkin and A.Yu Karseev, Optical Memory \& Neural Networks, (Information Optics) 23(3), 170-176 (2014)

20. V.V. Davydov, V.I. Dudkin and A.U. Karseev, Optical Memory \& Neural Networks (Information Optics) 22(2), 112-117 (2013)

21. V.V. Davydov, V.I. Dudkin and A. Yu. Karseev, Optical Memory \& Neural Networks (Information Optics) 23(4), 259-264 (2014)

22. V.V. Davydov, V.I. Dudkin, E.N. Velichko and A.Yu. Karseev, Journal of Optical Technology (A 
Translation of Opticheskii Zhurnal) 82(3), 132-135 (2015)

23. D.A. Klinov, A.V. Gulevich, V.S. Kagramanyan, V.M. Dekusar and V.D. Usanov, Atomic Energy 125(3), 143-148 (2019)

24. V.V. Ignatiev, M.Y. Kormilitsyn, L.A. Kormilitsyna, Yu.M. Semchenkov, Yu.S. Fedorov, O.S. Feinberg, A.V. Kruikov and A.V. Khaperskaya, Atomic Energy 125(5), 279-283 (2019)

25. V.G. Krapivtsev and V.I. Solonin, Atomic Energy 125 (5), 307-317 (2019)

26. I.I. Kryshev, L.A. Kurydina and I.I. Linge, Atomic Energy 117 (3), 159-163 (2014)

27. A.P. Elokhin, Atomic Energy 112 (4), 269-280 (2012)

28. A.P. Elokhin, Atomic Energy 117(3), 206-215 (2015)

29. V.V. Davydov, E.N. Velichko, V.I. Dudkin and A.Yu. Karseev, Measuring Techniques 57(6), 684689 (2014)

30. M.Y. Marusina, B.A. Bazarov, P.A. Galaidin, M.P. Marusin, A.A. Silaev, E.Y. Zakemovskya and Y.N. Mustaev, Measurement Techniques 57(5), 580-586 (2014)

31. V.V. Davydov, V.I. Dudkin and A.Yu. Karseev, Measurement Techniques 58(3), 317-322 (2015)

32. V.V. Davydov, V.I. Dudkin and N.S. Myazin, Journal of Communications Technology and Electronics 61(10), 1159-1165 (2016)

33. A.P. Elokhin and E.N. Kononov, Atomic Energy 80(2), 135-142 (1996)

34. M. Sivers, G. Fokin, P. Dmitriev, A. Kireev, D. Volgushev and A.-O.A.H. Ali, Lecture Notes in Computer Science 9870, 465-476 (2016)

35. R. Davydov, V. Antonov and N. Kalinin, Journal of Physics: Conference Series 643(1), 012107 (2015)

36. R.V. Davydov and V.I. Antonov, Journal of Physics: Conference Series 769(1), 012060 (2016)

37. R.V. Davydov and V.I. Antonov, Journal of Physics: Conference Series 929(1), 012040 (2017)

38. R.V. Davydov, V.I. Antonov and A.V. Moroz Proceedings of the 2018 IEEE International Conference on Electrical Engineering and Photonics, EExPolytech, 8564378 236-239 (2018)

39. V.V. Davydov, A.Yu. Karseev, E.K. Nepomnyashchay, A.A. Petrov and E.N. Velichko, Lecture Notes in Computer Science, 8638 694-702 (2014)

40. V.V. Davydov, N.V. Sharova, E.V. Fedorova, V.A. Vologdin and A.Yu. Karseev, Lecture Notes in Computer Science, 9247, 712-721 (2015)

41. V.A. Vologdin, V.V. Davydov and E.N. Velichko, Journal of Physics: Conference Series 741(1), 012095 (2016)
42. N.M. Grebenikova, K.J. Smirnov, V.V. Artemiev, V.V. Davydov, and S.V. Kruzhalov, Journal of Physics: Conference Series 1038(1), 012089 (2018)

43. A.S. Podstrigaev, R.V. Davydov, V.Yu. Rud' and V.V. Davydov, Lecture Notes in Computer Science 11118, 624-630 (2018)

44. R.V. Davydov, I.V. Saveliev, V.A. Lenets, M.Yu. Tarasenko, T.R. Yalunina, V.V. Davydov and V.Yu. Rud', Lecture Notes in Computer Science 10531, 177-183 (2017)

45. V.A. Lenets, M.Yu. Tarasenko, V.V. Davydov, N.S. Rodugina and A.V. Moroz, Journal of Physics: Conference Series 1038(1), 012037 (2018)

46. V.V. Sergeev, M.R. Petrichenko, D.V. Nemova, E.V. Kotov, D.S. Tarasova, A.V. Nefedova and A.B. Borodinecs, Magazine of Civil Engineering 84(8), 67-74 (2018)

47. V. Maslak, N. Nasonkina, V. Sakhnoskaya, S. Antonenko and D. Nemova, Procedia Engineering 117(1), 985-994 (2015)

48. V. Mushchanov, V. Sievka, A. Veshnevska and D. Nemova, Procedia Engineering 117(1), 1018-1026 (2015)

49. M. Petrichenko, N. Vatin, D. Nemova, N. Kharkov and A. Staritcyna, Applied Mechanics and Materials 627, 297-303 (2014)

50. V.V. Davydov, Measurement Techniques 59(11), 1202-1209 (2017) 